\title{
Simulation of space weathering of planet-forming materials: Nanosecond pulse laser irradiation and proton implantation on olivine and pyroxene samples
}

\author{
Maho Yamada ${ }^{1 *}$, Sho Sasaki ${ }^{1}$, Hiroko Nagahara ${ }^{1}$, Akira Fujiwara $^{2}$, Sunao Hasegawa ${ }^{2}$, Hajime Yano $^{2}$, \\ Takahiro Hiroi ${ }^{3}$, Hideo Ohashi ${ }^{4}$, and Hisashi Otake ${ }^{5}$ \\ ${ }^{1}$ Geological Institute, School of Science, University of Tokyo, Tokyo 113-0033, Japan \\ ${ }^{2}$ Institute of Space and Astronautical Science, Sagamihara, Kanagawa 229-8510, Japan \\ ${ }^{3}$ Department of Geological Sciences, Brown University, Providence, RI 02912, U.S.A. \\ ${ }^{4}$ Laboratory for Physics, Tokyo University of Fisheries, Minato-ku, Tokyo 108-0075, Japan \\ ${ }^{5}$ Tsukuba Space Center, National Space Development Agency of Japan, Ibaraki 305-8505, Japan
}

(Received December 1, 1998; Revised June 30, 1999; Accepted September 4, 1999)

\begin{abstract}
For the purpose of simulating the surface alteration process called "space weathering", experiments of pulse laser irradiation, proton implantation, and laser irradiation to proton implanted samples were performed and reflectance spectra of altered materials were measured. To simulate the impact heating by micrometeorite bombardments, we made a new apparatus using a pulse laser whose pulse duration is 6-8 nanoseconds, comparable with a timescale of micrometeorite impacts. We find that the degree of space weathering, i.e., change of reflectance spectrum should depend on mineral composition. Laser irradiation onto olivine produces the largest reduction of albedo and the highest reddening of reflectance spectrum. In general, variation of olivine spectra is much larger than that of pyroxenes. Depths of absorption bands do not change in the scaled spectra. The olivine spectrum after the laser irradiation can match spectra of some olivine asteroids within a subtype of S-type asteroids. Comparison of Vesta spectrum with altered pyroxene spectra suggests that Vesta surface would be relatively older than olivine asteroids. We also investigate the influence of solar wind proton and pyroxene $\mathrm{FeO}$ content. The proton implantation causes small changes in olivine and enstatite spectra. Implanted protons do not influence spectral change by the laser irradiation: the laser irradiation and the proton implantation do not produce multiplicative but additive changes on the reflectance spectra. FeO content of pyroxenes does not relate to the degree of reflectance change.
\end{abstract}

\section{Introduction}

The lack of spectral link between ordinary chondrites and asteroids is a long-standing problem. Some believe that S-type asteroids are parent bodies of ordinary chondrites (Chapman, 1996). But S-type asteroids are characterized by steep red continua unlike those of the ordinary chondrites, and their spectrally derived mineralogies are outside the range of ordinary chondrites. Gaffey et al. (1993) stated that S(IV) asteroids are mainly composed of pyroxene and olivine, which are consistent with chemical composition of ordinary chondrites. However, S(IV) asteroids have also redder reflectance slope than ordinary chondrites. The spectral mismatch between S-type asteroids and ordinary chondrites would be explained by so-called "space weathering" process where bombardments of micrometeorites altered the optical properties of the uppermost regolith of asteroids. Plausible processes such as impact-induced reduction of silicate iron, both in the impact melt and the impact-generated vapor, appear to produce optically important effects on the surface regolith. Recent observations by Galileo spacecraft indicate

* Now at Japan Space Forum, 1-29-6 Hamamatsu-cho, Minato-ku, Tokyo 105-0013, Japan.

Copy right(C) The Society of Geomagnetism and Earth, Planetary and Space Sciences (SGEPSS); The Seismological Society of Japan; The Volcanological Society of Japan; The Geodetic Society of Japan; The Japanese Society for Planetary Sciences. that there are optically different terrains on the surface of S-type asteroids Gaspra and Ida (Helfenstein et al., 1994, 1996): some craters and ejecta show less-altered reflectance spectrum explained by olivine-pyroxene mixture with a clear $1 \mu \mathrm{m}$ absorption band, whereas most of the surface has redder reflectance with a shallower absorption band.

Reflectance spectra of lunar soils have lower albedo, more reddened continuum, and weaker absorption bands than those of finely ground lunar rocks (e.g., Pieters et al., 1993). These are ascribed to the irradiation by micrometeorite impacts. Among lunar soils, the finest size fraction should control the weathered optical properties. Lower albedo would be mainly due to the agglutinate glasses in soils. But agglutinate glasses cannot explain the all feature of space weathering. Studies of lunar regolith fractions indicate that "mature" soils, containing more SMFe (submicroscopic metallic iron), are darker and somewhat redder than "immature" soils of the same composition, and features in the reflectance spectra become more subdued with the increasing soil maturity. The SMFe would be formed when soil particles containing solar wind protons are heated by micrometeorite impacts. A theoretical model calculated by Hapke (1993) demonstrates that SMFe distributed throughout the glass and also coating the crystalline grains can account for the optical properties of lunar soils. In fact, Keller and McKay (1997) found nanophase 
Fe as inclusions in lunar agglutinitic glasses and in thin rims surrounding lunar soil grains.

In simulating the irradiation by micrometeorite impacts, high-velocity $(>10 \mathrm{~km} / \mathrm{s})$ impact experiments can hardly produce enough number of dust impacts. Moreover, electrostatic dust accelerators, which can produce high velocity particles, should use conductive dust materials (e.g., C, Fe, Ag) which are not suitable to see the optical effects on target materials. Previously irradiation experiments were performed by continuous irradiation using a fusion furnace (Clark et al., 1992, Allen, 1993). Using a pulse laser for irradiation, Moroz et al. (1996) obtained results where ordinary chondrites were modified to have S-type characteristics. Wasson et al. (1998) heated HED meteorites by the same apparatus and observed the spectral change. However, the laser pulse duration was $0.5-1 \mu \mathrm{sec}$ which was 1000 times of real timescale of micrometeorite (1-10 $\mu \mathrm{m}$ size) impacts. Moreover, effects of protons and Fe contents have not been discussed in detail. In the present study of space weathering simulation, we used a pulse laser whose pulse duration is $6-8 \mathrm{nsec}$ (nanosecond), comparable with the timescale of micrometeorite impacts (Kissel and Krueger, 1987). We also implanted protons into sample materials to see effect of reduction of silicates.

\section{Experimental Procedure}

Figure 1 shows the overall procedure of our experiments. Mineral samples were comminuted into fine particles. We irradiated a pulse laser beam onto the sample to simulate the irradiation of micrometeoroid bombardments, or we implanted proton particles onto the sample to simulate the solar wind implantation. Then we irradiated a pulse laser onto some of proton-implanted samples. Finally we measured reflectance spectra of the processed samples and compare them with those of the comminuted raw samples.

\subsection{Sample materials}

We used olivine and orthopyroxene for the present experiments. Both are common in most meteorites such as ordinary chondrite. Olivine is a pure sample from San Carlos with $8.97 \mathrm{wt} \% \mathrm{FeO}\left(\mathrm{Fo}_{91}\right)$. In order to study the influence of iron content on the spectral change, we used several different pyroxenes. Enstatite from Bamble, Norway, is essentially a pure sample containing $9.88 \mathrm{wt} \% \mathrm{FeO}\left(\mathrm{En}_{85}\right)$. Pure hypersthene from Mäntyharju, Finland, contains $16.70 \mathrm{wt} \% \mathrm{FeO}$ $\left(E_{71}\right)$. Diogenite Tatahouine meteorite, from Tatahouine, Tunisia, is almost pure orthopyroxene whose composition is $\mathrm{En}_{75}$. Samples were ground with a pestle in an agate mortar and separated to a particle size $<75 \mu \mathrm{m}$ by a stainless steel sieve.

\subsection{Pulse laser irradiation}

In order to simulate the irradiation by micrometeorite impacts, powdered specimens were irradiated by a pulse laser beam under a vacuum at $2 \times 10^{-5} \mathrm{mmHg}$. We manufactured a new apparatus for this experiment to achieve a uniform two-dimensional scanning of the laser beam. The configuration of the device is shown in Fig. 2. A small chamber containing a sample holder is placed on an automatic X-Y stage. We used a solid-state Nd-YAG pulse laser with impulse frequency $20 \mathrm{~Hz}$. The wavelength of the laser was $1064 \mathrm{~nm}$. Pulse duration was 6-8 nsec, which is comparable with a real micrometeorite bombardment timescale (a few

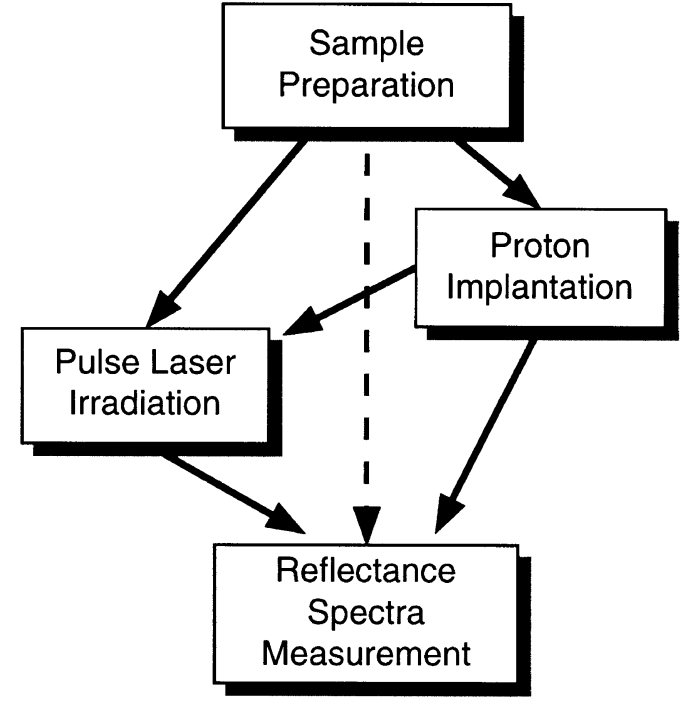

Fig. 1. Procedure in the present study. We measured reflectance spectra of raw samples, samples after the laser irradiation, samples after the proton implantation, and samples after the proton implantation and the laser irradiation.

times of the timescale where a shock wave by a micrometeorite of velocity $10 \mathrm{~km} / \mathrm{s}$ propagates through the particle size $10 \mu \mathrm{m} \sim 1 \mathrm{nsec})$. Pulse energy is variable from 1 to $30 \mathrm{~mJ}$. The diameter of the original laser beam was $3 \mathrm{~mm}$ and that of the focused beam is about $500 \mu \mathrm{m}$ on the sample surface. In the present system, the axis of the laser beam is fixed. To achieve uniform irradiation on the sample surface, the chamber is moved by the automatic X-Y stage with scanning velocity $5 \mathrm{~mm} / \mathrm{sec}$, allowing rather uniform irradiation on the sample surface (Fig. 2). The total irradiated energy in unit area were 8,120 and $240 \mathrm{~mJ} \mathrm{~mm}^{-2}$, for 1,15 , and $30 \mathrm{~mJ}$ pulse energy, respectively. In terms of energy deposition rate under $30 \mathrm{~mJ}$ pulse laser with $6 \mathrm{nsec}$ pulse width, we attain $10^{10} \mathrm{Wcm}^{-2}$, where evaporation and ion formation is expected (Kissel and Krueger, 1987). On the other hand, under microsecond pulse laser of comparable pulse energy, energy deposition rate is as low as $10^{7-8} \mathrm{Wcm}^{-2}$, beam energy is mostly absorbed to heat up the materials.

The powdered samples are placed in the aluminum holder. Weight of the powder is $0.10 \mathrm{~g}$ and thickness of the powder layer is about $1 \mathrm{~mm}$. For the irradiation target, we also used pellet samples of $15 \mathrm{~mm}$ diameter. Each pellet is surrounded by an aluminum ring. Powdered samples $(<75 \mu \mathrm{m})$ are packed into a pellet under 12 ton load. Using a pellet sample, we need not repack the irradiated particles before reflectance measurement. During the irradiation of the laser whose pulse energy is higher than $1 \mathrm{~mJ}$, we observed pressure increase in the chamber, suggesting the evaporation of target materials. Since powdered materials were sometimes scattered by the evaporated vapor, the holder floor could be irradiated by the laser and contamination from aluminum might take place. Therefore, for the irradiation on the powdered sample, we made a pellet of the same target materials and put it under the powered sample. 


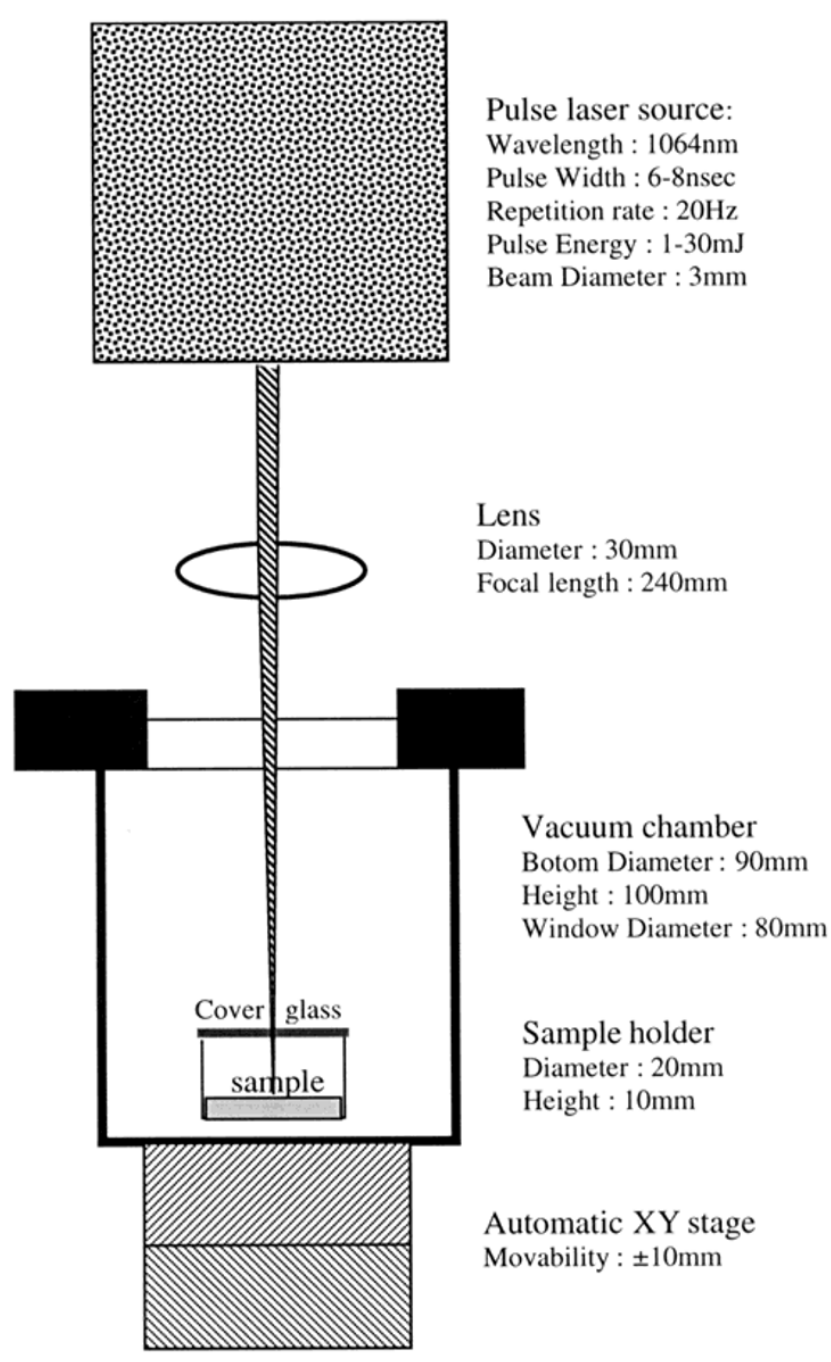

Fig. 2. Configuration of the laser irradiation system in the present experiment. A small chamber including the sample holder is placed on the automatic X-Y stage. We used a turbomolecular pump in the vacuum system to avoid contamination from oil vapor.

\subsection{Proton implantation}

We performed proton implantation experiments by RAPID (Rutherford Backscattering Spectroscopic Analyzer with Particle Induced X-ray Emission and Ion Implantation Devices) located at Research Center for Nuclear Science and Technology, the University of Tokyo. The resulting beam was about a few $\mathrm{mm}$ in diameter with energy $1 \mathrm{MeV}$ with a current density $J$ of about $15 \sim 30 \mathrm{nA} / \mathrm{cm}^{2}$ which varied depending on the machine condition. We chose the energy level so high that protons should be implanted firmly. The energy range of protons is within that of typical solar flare and much higher than that of normal solar wind. Although the total dose of normal solar wind particles is large and they could be effective in the surface sputtering, it would be doubtful that those particles should act as reducing agents.

The total dose $N_{d}$ of implanted atoms can be expressed by

$$
N_{d}=\frac{n}{e Z} \int_{0}^{T} J(t) d t
$$

where $n$ is number of atoms consisting of a charged particle and $Z$ is charge number of the particle $(n=1$ and $Z=1$ for the proton acceleration). In the present experiment, $N_{d}$ of these samples were $4.9 \times 10^{15}$ and $1.4 \times 10^{16} \mathrm{~cm}^{-2}$. Since the target surface of RAPID is vertical and ion beam comes horizontally with $7^{\circ}$ incident angle, we made a special sample holder which is covered with a carbon-coated Mylar film, preventing powders not only from falling but also from being charged up (Ohashi et al., 1993). Charge neutralization is necessary when the irradiated target is insulator, because it is easily charged up and no more protons could be injected. We doped graphite onto one side of a mylar film of $2.7 \mu \mathrm{m}$ thickness. This conductive film should be also useful in removing heat from the target materials. The powdered samples are packed into a holder whose depth is $1 \mathrm{~mm}$. After the proton implantation, the sample is removed and repacked into another holder for the reflectance measurement or for the laser irradiation.

\subsection{Measurements of reflectance spectra}

After the laser and/or proton irradiation, reflectance spectra of samples were measured. Bidirectional reflectance spectra were obtained at the facility at Future Space Systems Laboratory, at Tsukuba Space Center of National Space Development Agency of Japan (NASDA). Observational geometry was set up to simulate that of telescopic observation: an incidence angle of $30^{\circ}$ and an emission angle of $0^{\circ}$. Bidirectional reflectance spectra in the wavelength range 250-2600 $\mathrm{nm}$ were recorded at every $10 \mathrm{~nm}$. All spectra were measured relative to a spectralon, a near perfect diffuse reflector in this wavelength region. We confirmed that errors caused by sample packing were less than $2 \%$ in reflectance spectra. To see the spectrum change of the most affected sample surface, we also measured pellet samples which were not repacked after the laser irradiation.

Some of the products were also observed with JEOL 840 scanning electron microscope (SEM) equipped with LINK AN10000 energy dispersive spectrometer (EDS) installed at Geological Institute of University of Tokyo.

\section{Results}

\subsection{Reflectance spectra of laser irradiated samples Olivine}

Experiments of laser irradiation using olivine pellets were carried out under various pulse energy of 1, 15 and $30 \mathrm{~mJ}$. Figure 3(a) shows resulting spectra compared with that of a non-irradiated sample, and Fig. 3(b) shows normalized spectra at $560 \mathrm{~nm}$. As for the sample irradiated at $1 \mathrm{~mJ}$-pulse, no spectral change is observed all over the wavelength. The spectrum of irradiated sample at $15 \mathrm{~mJ}$-pulse has a striking drop of reflectance, especially in the ultraviolet and visible region. The maximum of the reduction is $34 \%$ at $410 \mathrm{~nm}$. At the highest pulse energy, $30 \mathrm{~mJ}$, the irradiated olivine sample shows more diminution of albedo. The maximum change of the reflectance is $46 \%$ at $420 \mathrm{~nm}$. Both spectra are reduced about $10 \%$ even at the region where wavelength is longer than $1500 \mathrm{~nm}$. In the raw spectra, the absorption around $1000 \mathrm{~nm}$ seems to be weaker at higher energy irradiation. But if we look at normalized spectra in Fig. 3(b), the most striking feature is the change of reflectance slopes in the infrared region: significant reddening of the spectra. Measured from the continuum slope, the relative depth of the absorption around $1000 \mathrm{~nm}$ is not changed largely. 
Fig. 3(a)

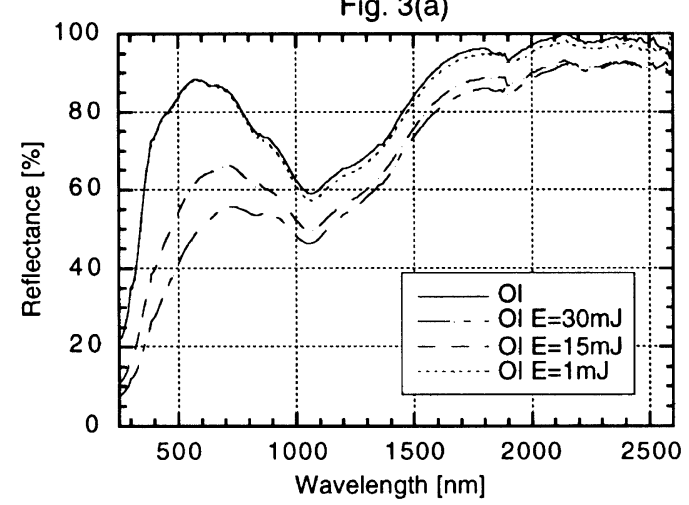

Fig. 3(c)

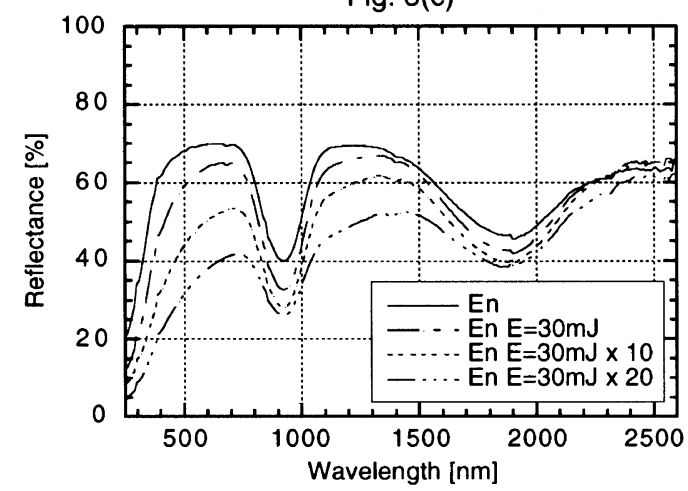

Fig. 3(e)

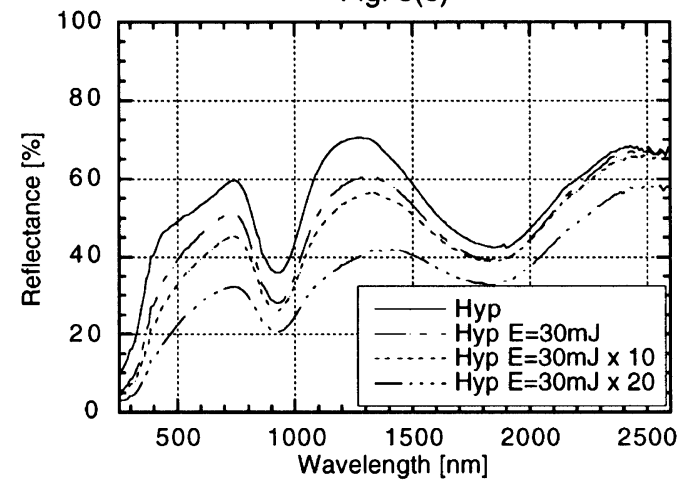

Fig. 3(b)

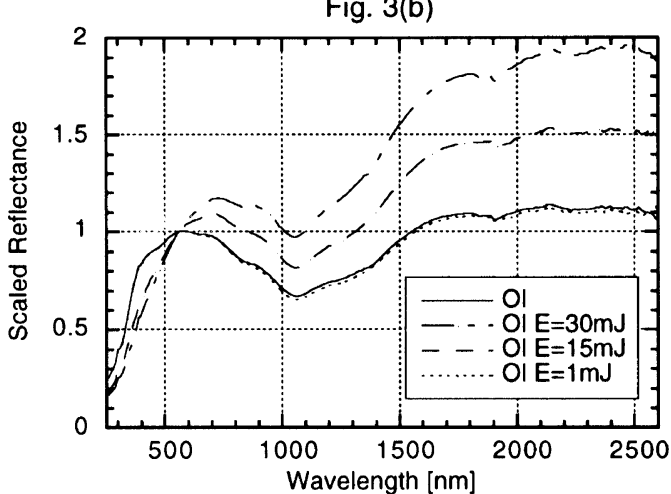

Fig. 3(d)

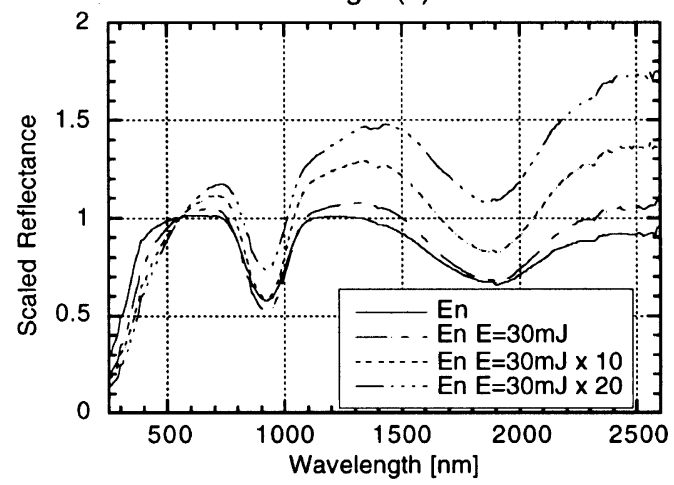

Fig. 3(f)

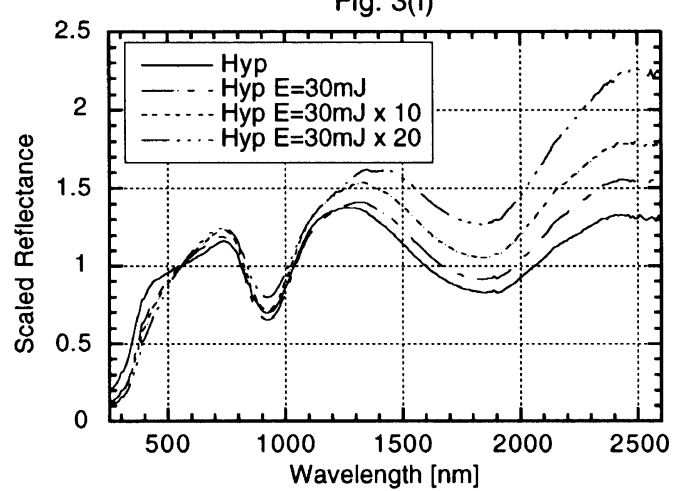

Fig. 3. Reflectance (UV-VIS-NIR) spectra of olivine and pyroxene pellet samples before and after the irradiation of pulse laser. (a) Absolute spectra of olivine. (b) Olivine spectra scaled at $560 \mathrm{~nm}$. There are four spectra (non-irradiated, irradiated under laser pulse energy $1,15 \mathrm{and} 30 \mathrm{~mJ}$ ). The sample irradiated by $1 \mathrm{~mJ}$-pulse shows no spectral change in overall wavelength. Spectra of samples irradiated at the energy of 15 and $30 \mathrm{~mJ}$ have a small peak of noise at $850 \mathrm{~nm}$. (c) Absolute spectra of enstatite. (d) Enstatite spectra scaled at $560 \mathrm{~nm}$. (e) Absolute spectra of hypersthene. (f) hypersthene spectra scaled at $560 \mathrm{~nm}$. Because of smaller spectral change than that of olivine, pulse laser irradiation was performed repeatedly at $30 \mathrm{~mJ}$ pulse energy. There are four spectra (non-irradiated, one irradiation, 10 times irradiations, and 20 times irradiations).

\section{Pyroxene}

Enstatite and hypersthene pellet samples were irradiated by the pulse laser at the energy of $30 \mathrm{~mJ}$. Resulting spectra are shown in Fig. 3(c) (enstatite) and Fig. 3(e) (hypersthene). Note that our raw enstatite sample contains $9.88 \% \mathrm{Fe}$ and the spectrum of the unprocessed sample has clear absorption bands. As for enstatite, after once $30 \mathrm{~mJ}$ laser irradiation, albedo at $370 \mathrm{~nm}$ decreases by $16 \%$, which is the maximum of reduction. Although reflectance decrement at $1170 \mathrm{~nm}$ of $17 \%$ is noticeable, little changes are observed near 1500 $\mathrm{nm}$ and beyond $2200 \mathrm{~nm}$. The reflectance of irradiated hypersthene is a little lowered at the wavelength smaller than
$2000 \mathrm{~nm}$. Darkening at $400 \mathrm{~nm}$ is $13 \%$ and notable decrease from $1100 \mathrm{~nm}$ to $1200 \mathrm{~nm}$ of about $12 \%$ is found. In result, changes of both spectra of pyroxene are much smaller than that of olivine. In order to see the effect of more irradiation, we performed 10 and 20 times repeated scannings of the laser irradiation onto pyroxene samples. Significant decreases in the reflectance are observed in the shorter wavelength $(<1500 \mathrm{~nm})$ regions of both samples, although the decrease is not proportional to the irradiation time. As seen in the normalized pattern (Figs. 3(d) and 3(f)), the spectra after the repeated irradiation show reddening in the infrared region. On the other hand, absorption band features around 
Fig. 4(a)

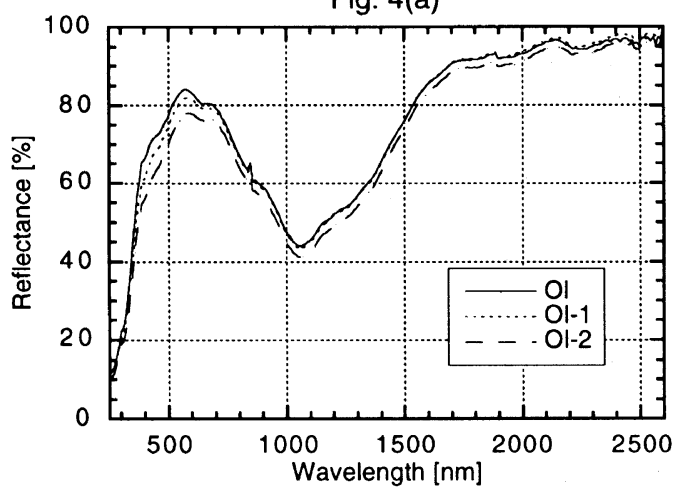

Fig. 4(c)

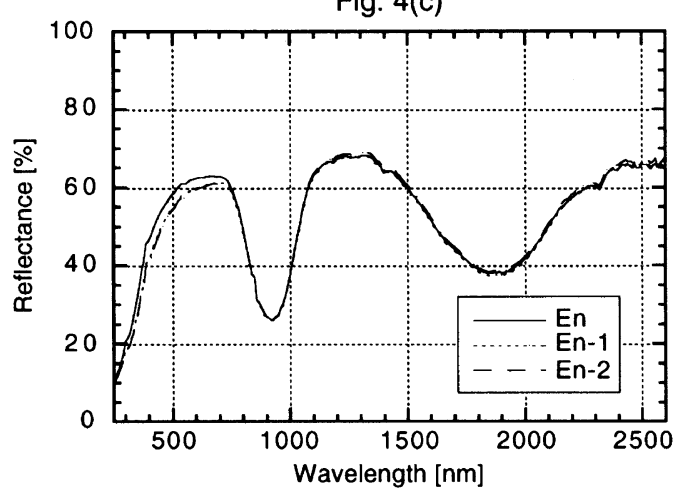

Fig. 4(e)

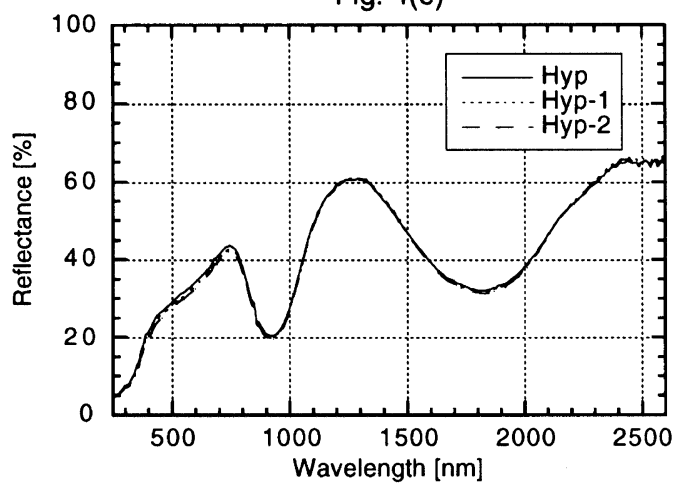

Fig. $4(g)$

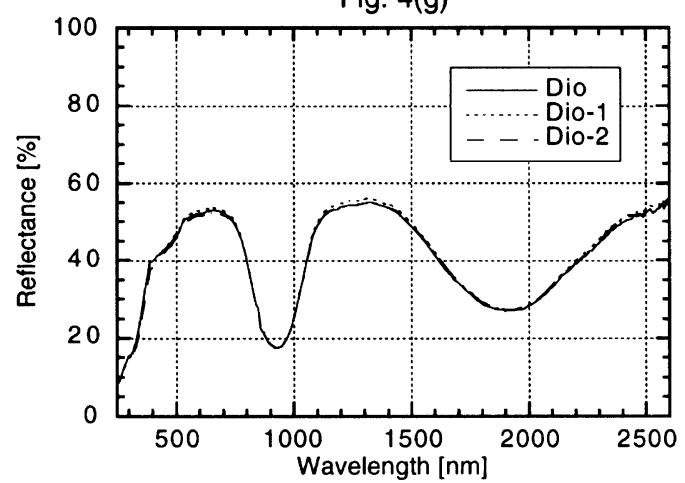

Fig. 4(b)

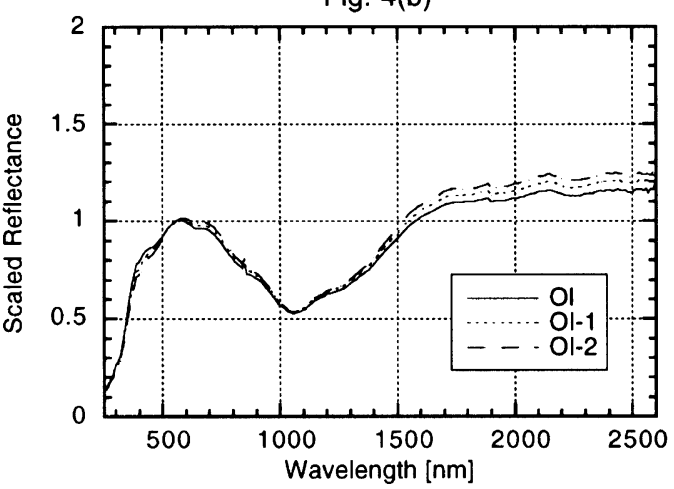

Fig. 4(d)

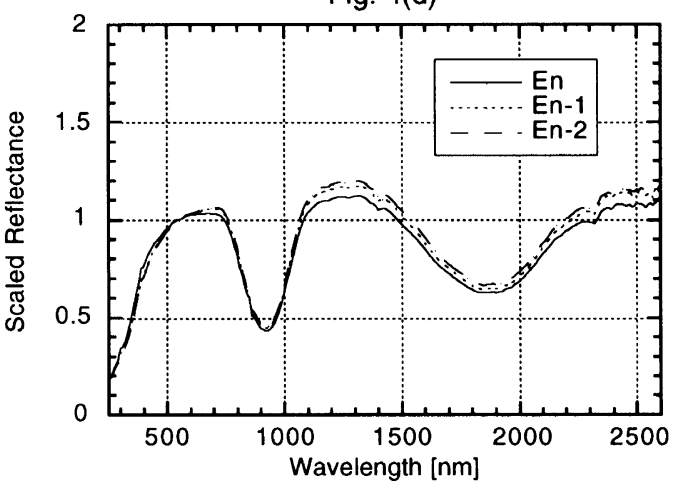

Fig. 4(f)

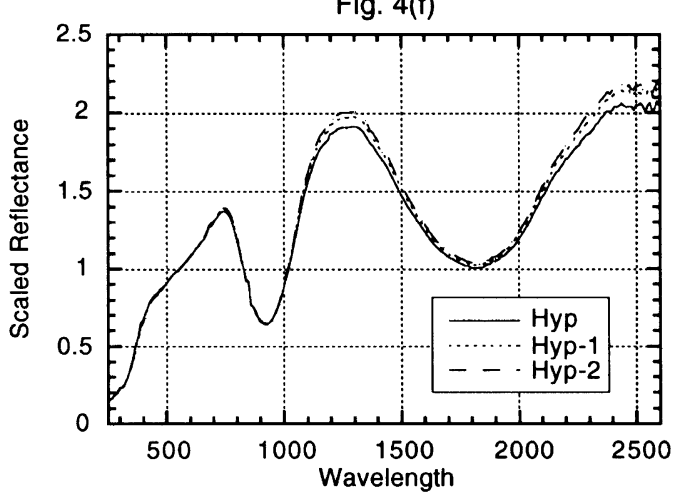

Fig. 4(h)

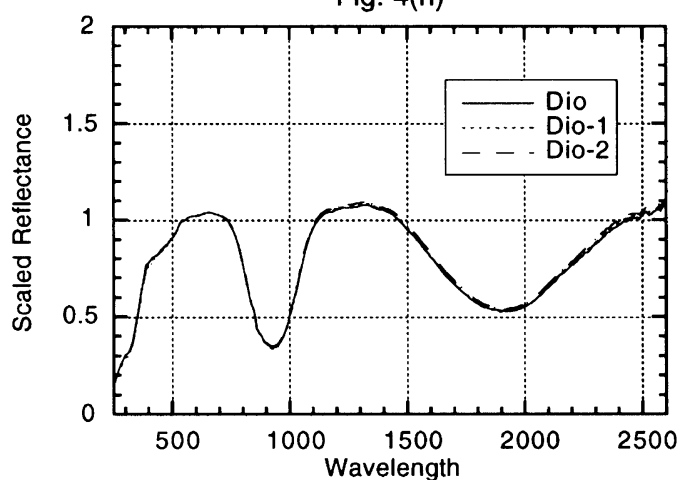

Fig. 4. Reflectance spectra of olivine and pyroxene powder samples before and after the implantation of high energy protons. Number 1 denotes the case of shorter irradiation whose total dose is $N_{d 1}=4.9 \times 10^{15} \mathrm{~cm}^{-2}$. Number 2 corresponds to the case of longer irradiation whose total dose is $N_{d 2}=1.4 \times 10^{16} \mathrm{~cm}^{-2}$. Reflectance of an unprocessed powder sample is usually lower than that of a pellet sample. (a) Absolute spectra of olivine. (b) Olivine spectra scaled at $560 \mathrm{~nm}$. (c) Absolute spectra of enstatite. (d) Enstatite spectra scaled at $560 \mathrm{~nm}$. (e) Absolute spectra of hypersthene. (f) hypersthene spectra scaled at $560 \mathrm{~nm}$. (g) Absolute spectra of diogenite. (h) Diogenite spectra scaled at $560 \mathrm{~nm}$. 
Fig. 5(a)

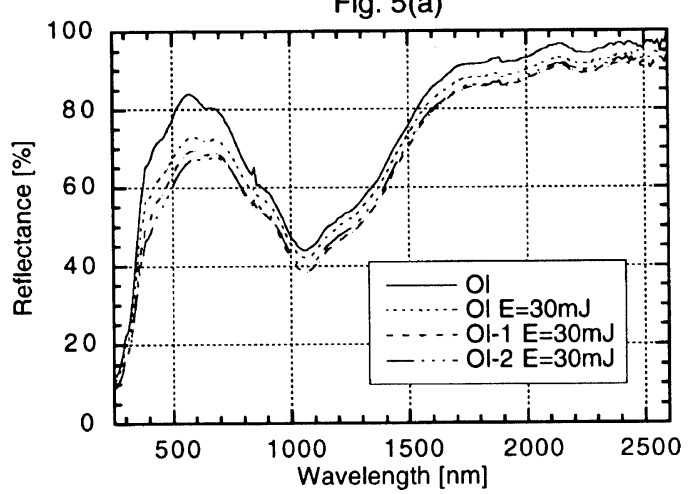

Fig. 5(c)

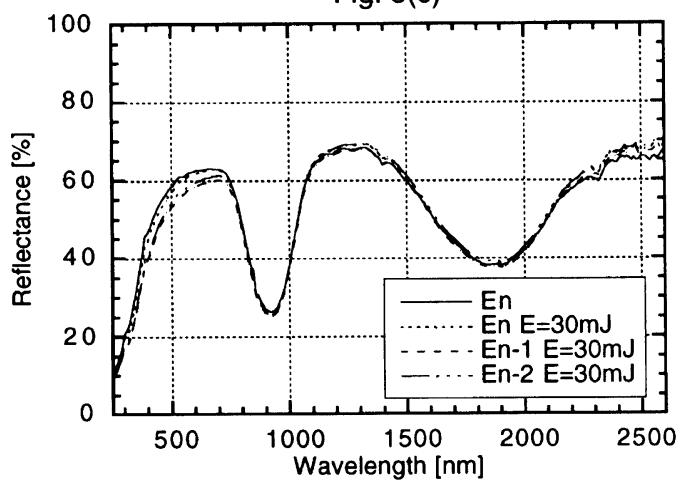

Fig. $5(\mathrm{e})$

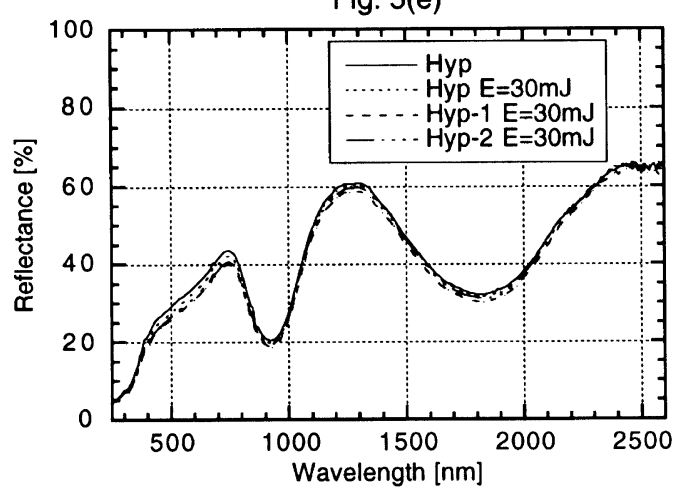

Fig. $5(\mathrm{~g})$

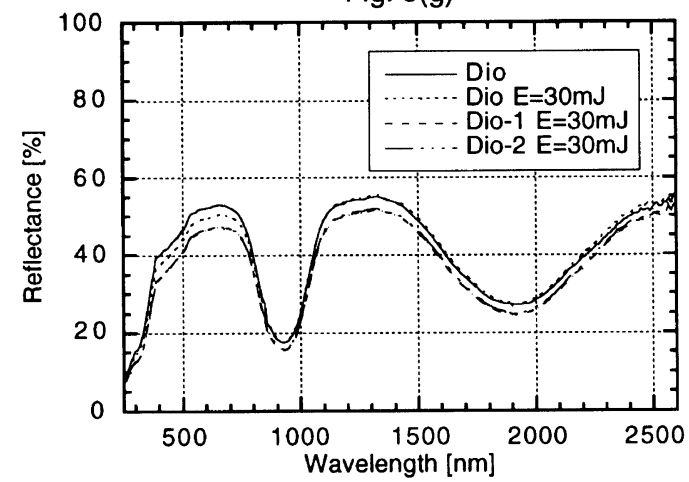

Fig. 5(b)

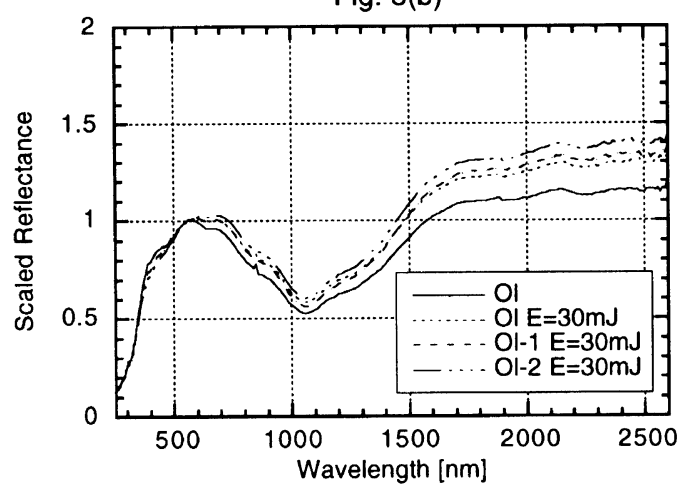

Fig. 5(d)

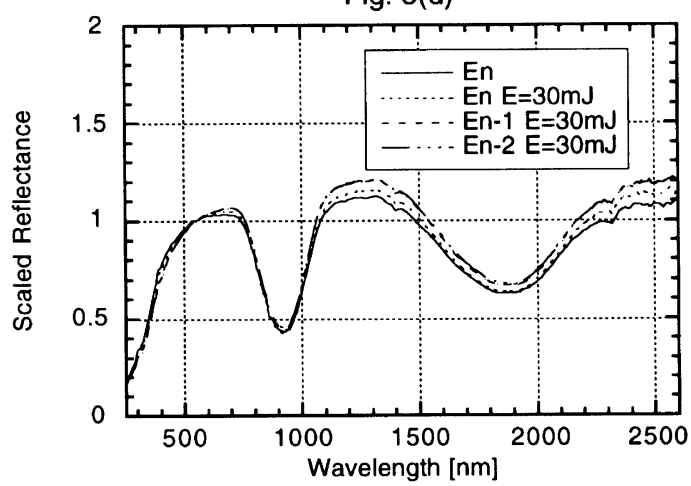

Fig. 5(f)

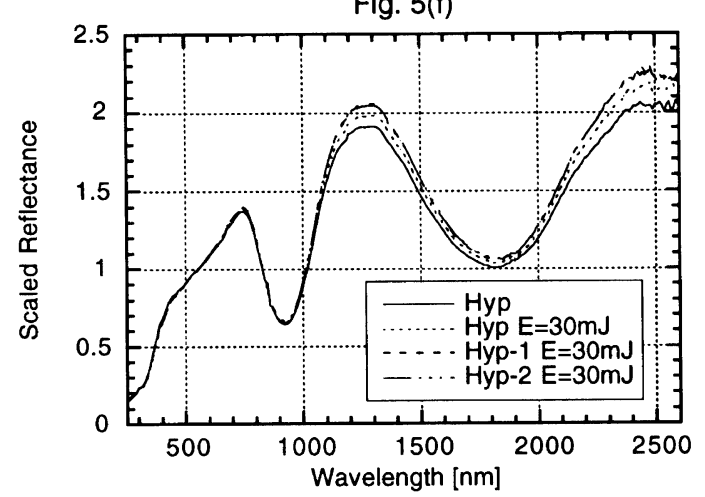

Fig. 5(h)

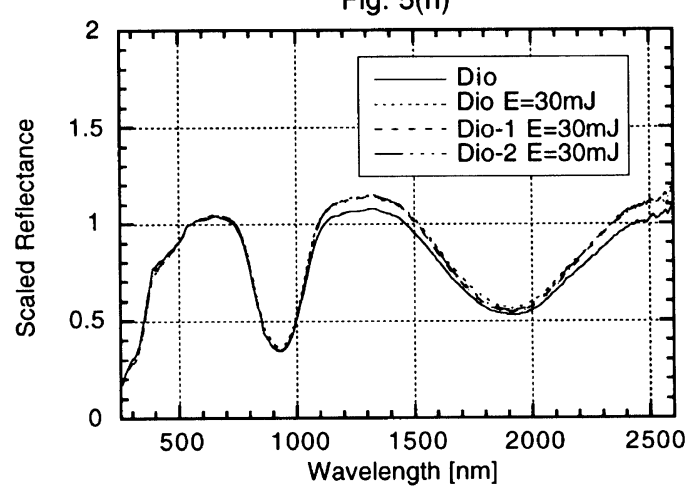

Fig. 5. Reflectance spectra of olivine and pyroxene powder samples after laser irradiation as well as proton implantation. Spectra of unprocessed powder samples and laser-irradiated samples without proton implantation are shown for comparison. (a) Absolute spectra of olivine. (b) Olivine spectra scaled at $560 \mathrm{~nm}$. (c) Absolute spectra of enstatite. (d) Enstatite spectra scaled at $560 \mathrm{~nm}$. (e) Absolute spectra of hypersthene. (f) hypersthene spectra scaled at $560 \mathrm{~nm}$. (g) Absolute spectra of diogenite. (h) Diogenite spectra scaled at $560 \mathrm{~nm}$. 
$1 \mu \mathrm{m}$ and $2 \mu \mathrm{m}$ are not largely changed. Normalized spectra show little change in depth and width of absorption bands. Although hypersthene contains $70 \%$ more FeO than enstatite, the results do not show significant dependence on $\mathrm{FeO}$ abundance of target materials.

\subsection{Reflectance spectra of proton-implanted samples}

Figure 4 shows the albedo changes of proton-implanted samples. Subscript 1 denotes the case of shorter proton irradiation whose total dose is $N_{d 1}=4.9 \times 10^{15} \mathrm{~cm}^{-2}$. Subscript 2 corresponds to the case of longer proton irradiation whose total dose is $N_{d 2}=1.4 \times 10^{16} \mathrm{~cm}^{-2}$. Reflectance of an unprocessed powder sample is usually lower than that of a pellet sample.

Olivine

Albedo changes of olivine after the proton implantation are significant (Fig. 4(a)). A sample after the shorter irradiation $\left(\mathrm{Ol}-1\right.$ at $\left.N_{d 1}=4.9 \times 10^{15} \mathrm{~cm}^{-2}\right)$ shows no difference from the unprocessed olivine at the wavelength longer than $700 \mathrm{~nm}$. It shows reduction of albedo from $300 \mathrm{~nm}$ to 700 $\mathrm{nm}$. The largest reduction is observed at $390 \mathrm{~nm}$ of $5 \%$. On the other hand, reflectance of olivine with more implanted proton $\left(\mathrm{Ol}-2\right.$ at $N_{d 2}=1.4 \times 10^{16} \mathrm{~cm}^{-2}$ ) decreases all over the measured wavelength range. In the region longer than $1500 \mathrm{~nm}$, changes are about $2 \%$. In ultraviolet and visible region, the reduction is apparently significant and decrease by $10.5 \%$ is recorded at $390 \mathrm{~nm}$. The scaled reflectance spectra are shown in Fig. 4(b). Although the pattern in ultraviolet and visible region are not changed largely, irradiated samples show an appreciable reddening in the infrared region $(>1000 \mathrm{~nm})$.

Pyroxene

Reflectance spectra of proton-implanted pyroxenes are shown in Figs. 4(c) to (h). As seen in Fig. 4(c), change of reflectance of enstatite is appreciable only at the wavelength shorter than $750 \mathrm{~nm}$. In the case of the small implantation dose (En-1 at $N_{d 1}$ ), reflectance decrease is $5.2 \%$ at $380 \mathrm{~nm}$. The result after the larger implantation dose (En-2 at $N_{d 2}$ ) resembles that of En-1 and it shows $6 \%$ reduction at $380 \mathrm{~nm}$. The difference between En-1 and En-2 is small. As seen in Figs. 4(e) and (g), reflectance changes of hypersthene and diogenite samples are only less than $2 \%$. We cannot say that those spectra are altered by proton implantation, although visible reflectance of hypersthene is slightly decreased. We did not see appreciable spectral change in diogenite samples. As a whole, similar to the pulse laser irradiation, spectral changes of pyroxene samples are much smaller than those of olivine samples. The scaled reflectance spectra (Figs. 4(b), (d), and (f)) show relative increase in the infrared reflectance. But this is simply due to a small decrease of the absolute reflectance in the visible wavelength.

\subsection{Proton-implanted and laser-irradiated samples}

In order to see whether the existence of hydrogen atoms may affect spectral alteration when minerals are heated by micrometeorite impacts, powder samples after the proton implantation were irradiated by the pulse laser whose pulse energy is $30 \mathrm{~mJ}$. We also irradiate pulse laser on the powder sample without the proton implantation. Results are shown in Figs. 5(a) to (h). In these experiments of powder samples, spectral changes of laser irradiated samples are smaller than those of pellet samples (Fig. 3).

\section{Olivine}

As seen in Figs. 5(a) and (b), after the pulse laser irradiation, the olivine sample without hydrogen implantation shows reflectance change only $2 \sim 3 \%$ in the near infrared region. Greater alteration of reflectance is seen in the visible region. Reduction is about $10 \%$ at $430 \mathrm{~nm}$ and $12 \%$ at $560 \mathrm{~nm}$. Proton-implanted samples (Ol-1 and Ol-2) after the laser irradiation show larger reduction. They are darkened at all measured wavelength by more than $5 \%$. Ol-1 after the laser irradiation shows reflectance reduction of $15 \%$ at 420 $\mathrm{nm}$ and of $15 \%$ at $560 \mathrm{~nm}$. Ol-2 after the laser irradiation shows reductions of $19 \%$ at $420 \mathrm{~nm}$ and of $18 \%$ at $560 \mathrm{~nm}$. Although the laser irradiation decreases the reflectance of proton-implanted olivine, the resulting reduction seems to be additive: spectral change by the pulse laser irradiation was not enhanced by the presence of hydrogen.

\section{Pyroxene}

Proton-implanted pyroxene samples were irradiated by the pulse laser at $30 \mathrm{~mJ}$. As seen in Fig. 5, the change of reflectance is smaller than that of olivine in general. As for enstatite samples in Figs. 5(c) and (d), En-1 and En-2 after the laser irradiation are slightly darkened; reductions of reflectance are $7 \%$ and $6.2 \%$, respectively, at $380 \mathrm{~nm}$. Decrease in reflectance from $300 \mathrm{~nm}$ to $600 \mathrm{~nm}$ is more than $4 \%$. However, spectral differences from the samples before the laser irradiation (Figs. 4(c) and (d)) are small and not larger than the difference between raw (En) and laser irradiated (En $\mathrm{E}=30 \mathrm{~mJ}$ ) samples in Figs. 5(c) and (d). Therefore, there is no multiplicative effect between the proton implantation and the laser irradiation. Also in hypersthene samples (Figs. 5(e) and (f)), we can only see the additive effect between the proton implantation and the laser irradiation. Figures $5(\mathrm{~g})$ and (h) show that diogenite is changed by the pulse laser irradiation in the visible region by more than $2 \%$. Proton implanted diogenite samples show difference more than $4 \%$. The maximum of them is $6 \%$ at $400 \mathrm{~nm}$ for both Dio- 1 and Dio- 2 . The laser irradiation would have decreased the reflectance of proton-implanted samples more than that without proton implantation. However, there is no difference between the scaled reflectances. The difference in the original spectra should be ascribed to the different relative amount of irradiated grains in the powder samples.

\section{Discussion}

\subsection{Dependence on proton implantation}

To investigate the effect of the solar wind particles as a reducer in the "space weathering" hypothesis, we carried out the proton implantation experiments. Although the actual energy of solar wind protons is about a Kev/amu, we chose higher energy $1 \mathrm{MeV}$ to inject protons steadily and deeper. Particles with energy of about some $\mathrm{KeV}$ act as sputtering source (Johnson and Baragiola, 1991). Their penetration depth is smaller than $0.1 \mu \mathrm{m}$ in lunar materials (Vaniman et al., 1991). On the other hand, protons of $\mathrm{MeV}$ energy range can penetrate on the order of $1 \mathrm{~mm}$. This energy corresponds to that of solar flare particles. The flux of solar wind on the surface of the moon is about $3 \times 10^{8} \mathrm{~cm}^{-2} \mathrm{sec}^{-1}$ (Vaniman et $a l ., 1991)$. The total dose in our experiments is equivalent to that of several months, or several tens of years even if using the flux of flare particles. 
As seen in Figs. 4 (a) to (d), we observed small spectral change in olivine and enstatite. Change of enstatite reflectance was limited in the visible wavelength. Similar to the laser irradiation experiments, olivine is more easily darkened than enstatite. Since the difference between En1 and En-2 is small, more reduction of visible reflectance would be doubtful even if more protons were implanted. We observed a very small change in hypersthene and no appreciable change in diogenite reflectance. Proton implantation only may not have changed reflectance to such a significant level as advocated by the process of "space weathering".

Recently Dukes et al. (1999) performed KeV range proton and helium bombardment on olivine surface. The total particle doses in their experiments are from $10^{16}$ to $10^{18} \mathrm{~cm}^{-2}$, somewhat larger than that of our experiments. Using in situ X-ray photoelectron spectroscopy, they found evidence of reduction of iron oxide, especially on the irradiation of helium atoms. However, no significant changes in reflectance spectra were obtained. They ascribed this to the reoxidation of iron after removal from the vacuum. Our experiments of higher energy $(\mathrm{MeV})$ proton implantation produced small but appreciable spectrum changes even under the smaller total dose. Further experiments supplying more proton dose would be necessary to discuss the extent of spectral change by the proton implantation in detail.

We also performed the pulse laser irradiation onto protonimplanted samples (Fig. 5). According to "space weathering" hypothesis, it is expected that the degree of alteration of a proton-implanted sample would be greater than that of the sample without implantation. In fact, olivine and pyroxene samples show such tendency. After the pulse laser irradiation, proton-implanted samples show more change than samples without protons. Samples containing more protons show redder spectra (Fig. 5). However, comparison between Fig. 4 and Fig. 5 suggests that the spectrum change due to the laser irradiation does not depend on the number of implanted protons. The spectrum change by the laser irradiation is not multiplicative but additive to the change by proton implantation; we find no evidence that implanted proton should enhance the spectrum change at pulse laser irradiation. In pyroxene samples, the change of hypersthene and diogenite samples would have been larger than that of enstatite if space weathering degree is proportional to $\mathrm{FeO}$ content. As seen in Figs. 4 and 5, hypersthene and diogenite samples show no more change than enstatite; we observed no dependence on FeO abundance.

\subsection{Dependence on laser energy}

The dependence on laser pulse energy was investigated using pellets of olivine. In Fig. 3, the higher the pulse energy becomes, the larger alteration occurs. They also show a remarkable reddening tendency. Albedo of olivine before and after the irradiation under the energy of $30 \mathrm{~mJ}$ changed from $88 \%$ down to $47 \%$, which is the largest alteration among all samples. The reflectance of pyroxenes also decreased, although the variation range is less than that of olivine and the dependence on $\mathrm{FeO}$ abundance is not observed, either.

We did not observe very large spectrum decrease such as Clark et al. (1992) and Moroz et al. (1996). In Moroz et al. (1996), olivine albedo changed from $73 \%$ to $24 \%$. Their experimental condition of laser energy was different from ours.
The total energy deposited per unit area of our experiments was $240 \mathrm{~mJ} \mathrm{~mm}^{-2}$ for $30 \mathrm{~mJ}$ pulse energy. Although the accurate total energy in Moroz et al. (1996) was unknown, it should be orders of magnitude larger than ours, judging from the statement that laser power was about $1.2 \mathrm{KW}$, the rate of rotation of sample was two revolutions per minute, and the impulse frequency of the apparatus was $30-40 \mathrm{kHz}$. Also the pulse duration in their experiments was $1 \mu \mathrm{sec}$, which is much longer than ours. Their experimental condition would be close to continuous irradiation, leading to the formation of melted glass.

In our experiments, most part of samples survived as undamaged minerals and clear evidence of vitrification was not found in the processed samples. Observation under a microscope, the altered samples contain brown rough-surfaced grains. This corresponds to the reduction of visible albedo after the pulse laser irradiation. Backscattered electron images (BEIs) of olivine pellet samples are shown in Fig. 6. The sample after the irradiation of $1 \mathrm{~mJ}$ pulse laser (Fig. 6(b)) shows no significant difference from the image of the unprocessed sample (Fig. 6(a)). This is compatible with the result of reflectance spectra: the sample after $1 \mathrm{~mJ}$ irradiation shows little change. On the other hand, the sample after the irradiation of $30 \mathrm{~mJ}$ pulse laser (Fig. 6(c)) shows different feature from the unprocessed sample. Small fragmentary particles between larger grains are lost probably due to scattering during the irradiation. Micron-sized holes are observed on the grain surface. These may have been formed by the surface evaporation. The sample after the twice irradiation of $30 \mathrm{~mJ}$ laser (Fig. 6(d)) shows a more subdued feature. Sharpness of grain edges is lost. There are larger surface holes. Overall feature would be due to progressive evaporation or to sintering among grains. EDS measurements detect no difference of chemical composition between raw and irradiated samples. Preliminary analysis of $30 \mathrm{~mJ}$ irradiated olivine grains by a transmission electron microscope also shows some evaporated or sintered feature.

Although we have not detected reduced iron, there is a possibility that the micro-scale evaporation could be responsible for the surface alteration causing the change of reflectance spectra. In fact, nanophase iron particles were found in the lunar soil rim, which could be formed by vapor deposition (Keller and McKay, 1997). Formation of nanophase iron particles might explain the difference of space weathering degree in our experiments. Since iron diffusion in olivine is much faster than that in pyroxene (Hiroi and Takeda, 1991), nanophase iron particles would be more enriched in olivine under the same heating condition. We have not seen any evidence of glass of melt, which was observed by Moroz et al. (1996). The cause of spectral change in our experiments might be different from lunar samples where fine glass particles probably from melts would control the darkening as well as the smearing of absorption bands. In effect, relative depth of $1 \mu \mathrm{m}$ absorption bands of olivine and pyroxene was not largely decreased in our experiments.

We also analyzed the altered samples after the pulse laser irradiation by X-ray diffraction. Minerals have characteristic reflection peaks corresponding to the atomic distance in its structure. If the systematic alteration we observed is dependent on crystal structure, intensity of particular peaks would 


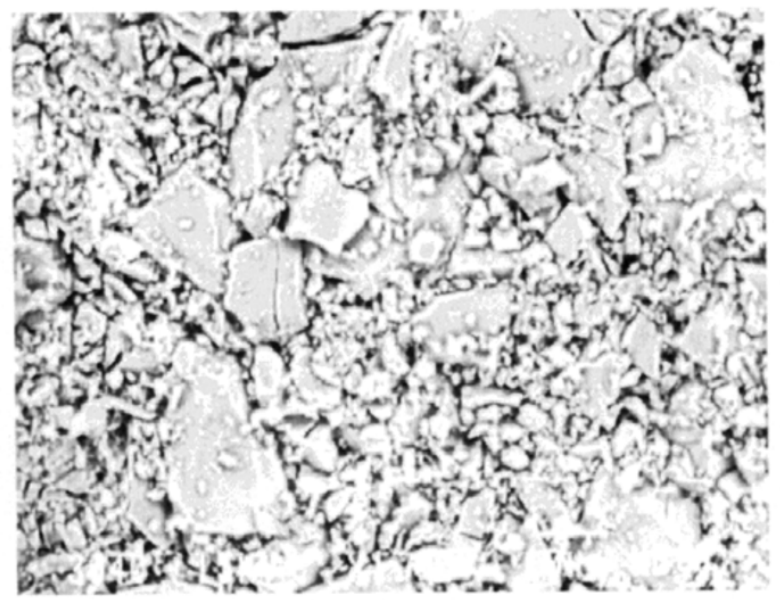

(a) Raw

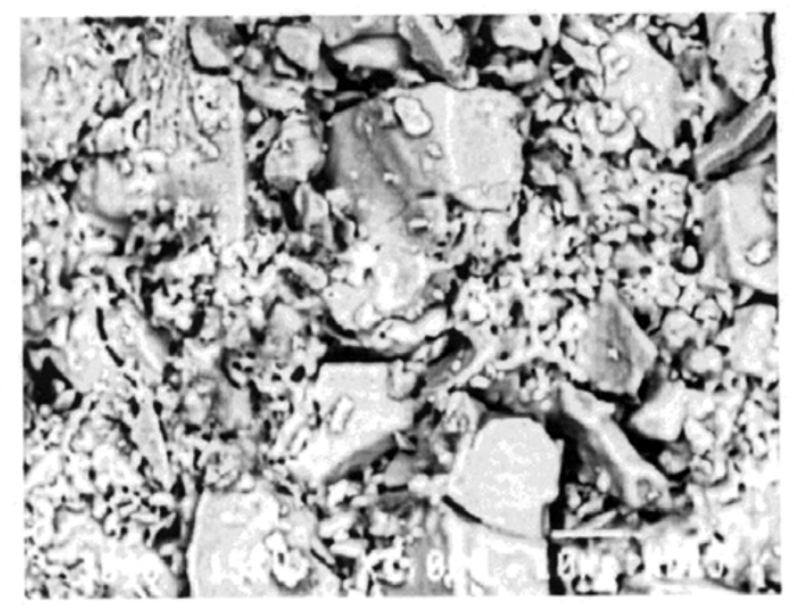

(c) $30 \mathrm{~mJ}$

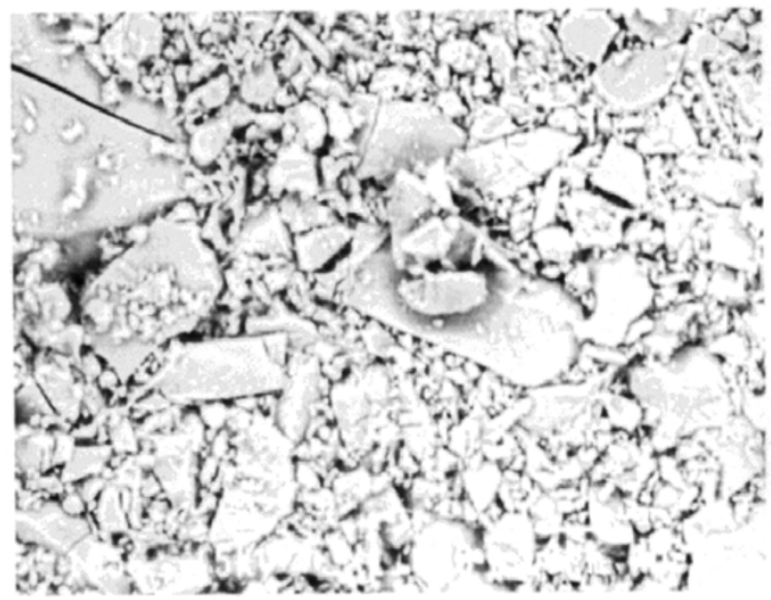

(b) $1 \mathrm{~mJ}$

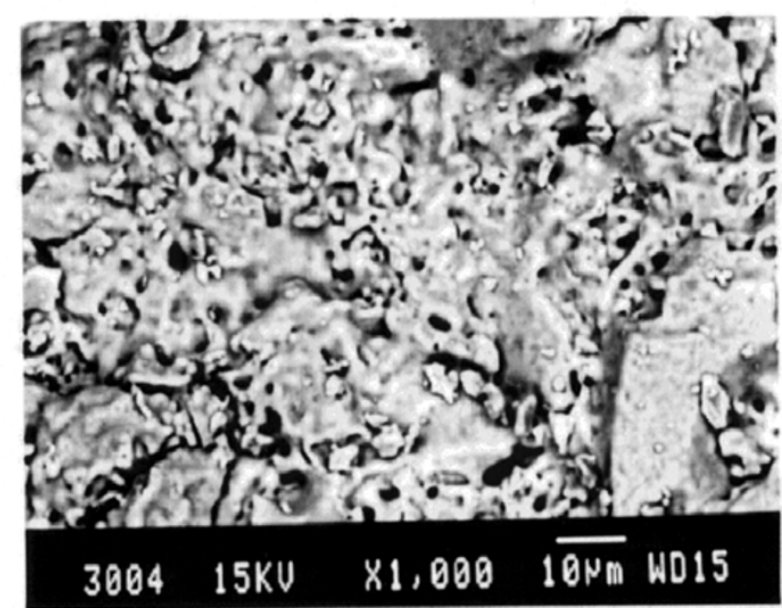

(d) $30 \mathrm{~mJ} \times 2$

Fig. 6. Backscattered electron images (BEI) of olivine pellet samples. All four images have the same magnification and the scale in the bottom denotes 10 $\mu \mathrm{m}$. (a) Unprocessed sample. (b) Sample after the irradiation of pulse laser whose pulse energy is 1 mJ. (c) Sample after the irradiation of pulse laser whose pulse energy is $30 \mathrm{~mJ}$. (d) Sample after the twice irradiation of pulse laser whose pulse energy is $30 \mathrm{~mJ}$.

be changed. Or apparent vitrification would make the peaks broaden. In results, we observed no systematic variation of peak intensity or half width in the diffraction spectra of irradiated samples. In addition, the peak of iron at $d=2.03 \AA$ was not observed. However, since a bulk powder sample is used in X-ray diffraction measurement, there would be possibility that altered surface particles played little role on the change of diffraction spectra.

\subsection{Comparison with asteroid spectra}

In Fig. 7(a), the reflectance spectrum of olivine after the irradiation of pulse laser at $30 \mathrm{~mJ}$ is compared with the spectra of two asteroids which are classified as olivine asteroids. They belong to a subset of S-type asteroids (Gaffey et al., 1993). Both asteroids show significant reddening of infrared spectra, which are well reproduced by the spectrum of laser irradiated sample. Feature of $1 \mu \mathrm{m}$ band is similar. Moreover, relatively flat feature above $2000 \mathrm{~nm}$ is well reproduced.

In Fig. 7(b), the spectrum of Vesta is compared with our results of laser-irradiated enstatite. Vesta has two clear ab- sorption bands ( $1 \mu \mathrm{m}$ and $2 \mu \mathrm{m}$ ) which are typical of pyroxenes. It is considered as the parent body of HED achondrites. Among our results, reflectance spectra of enstatite samples after repeated irradiations of $30 \mathrm{~mJ}$ pulse laser are most similar to the Vesta spectrum. After the repeated laser irradiations, the visible spectrum of enstatite has been declined from the relatively flat raw spectrum. Although the overall spectrum has a reddening feature, absorption band depths are not changed after the repeated irradiations.

Our results show that the reflectance of olivine should be much more easily changed than that of pyroxenes and that absorption band depths are not changed largely even after the repeated irradiation. Although the spectrum of Vesta has clear absorption bands, Vesta surface is not necessarily young. If the frequency of pulse laser irradiation corresponds to the relative timescale in the space, Vesta would be one order of magnitude older than the above olivine asteroids. Here we tentatively compare energy and frequency of irradiated pulse laser with the actual timescale in space. In our exper- 


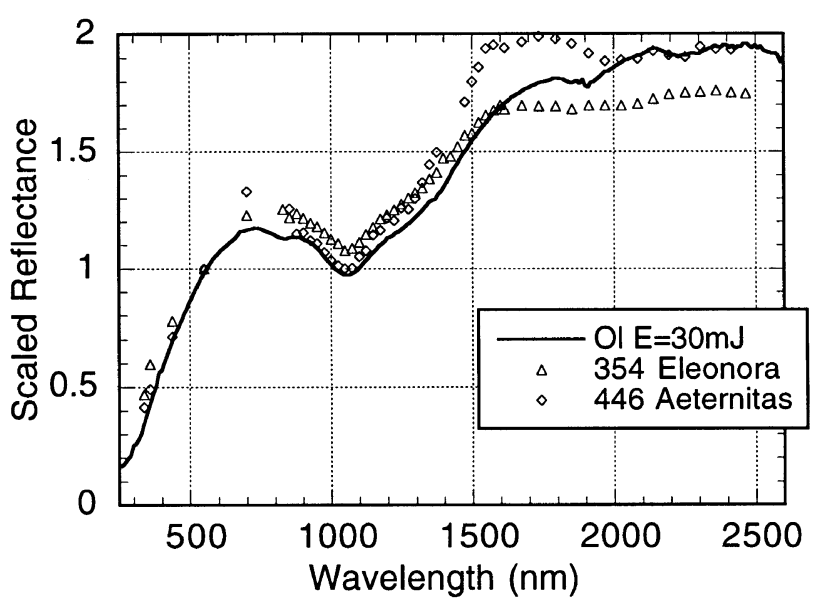

(a)

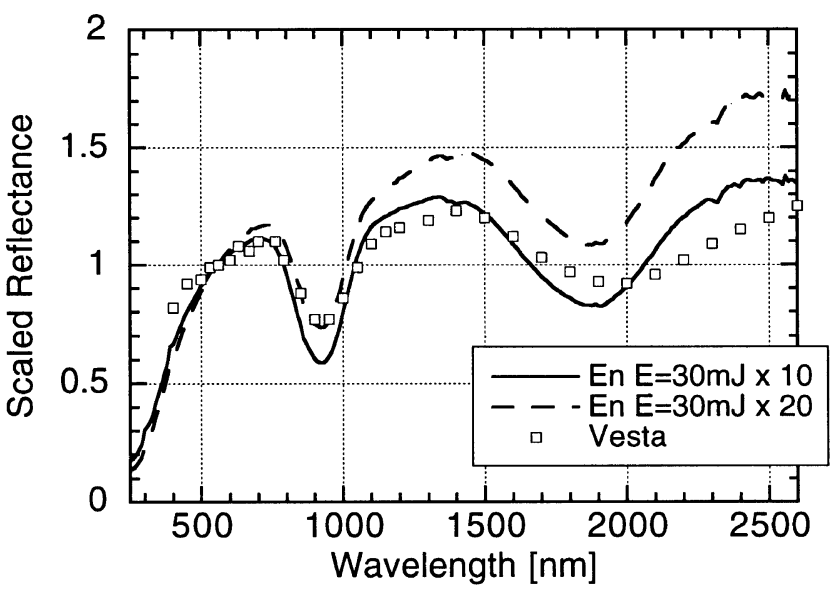

(b)

Fig. 7. Comparison of reflectance spectra after the pulse laser irradiation with asteroid spectra. (a) A scaled olivine spectrum after 30 mJ irradiation and the spectra of olivine asteroids Eleonora and Aeternitas (Gaffey et al., 1993). (b) Scaled pyroxene spectra after 10 and 20 times repeated irradiation at $30 \mathrm{~mJ}$ and the spectrum of Vesta.

iments, energy deposition at $30 \mathrm{~mJ}$ pulse laser irradiation is $2.4 \times 10^{5} \mathrm{Jm}^{-2}$. In space, impact rate of dust particles around $10^{-12} \mathrm{~g}(1 \mu \mathrm{m}$ in diameter $)$ is about a few $10^{-4} \mathrm{~m}^{-2} \mathrm{~s}^{-1}$ at 1 AU (Grün et al., 1991). One dust particle loses energy $2 \times 10^{-7} \mathrm{~J}$ at the impact with velocity $20 \mathrm{~km} / \mathrm{s}$. Then, the total energy deposition rate by dust impacts is about $10^{-3}$ $\mathrm{mJ} \mathrm{m} \mathrm{m}^{-2} \mathrm{yr}^{-1}$. Supposing a simple comparison between the irradiation energy by pulse laser and released energy at dust impacts, irradiation of $30 \mathrm{~mJ}$ pulse laser in our experiments corresponds to a few $10^{8} \mathrm{yr}$ in the space. This might correspond to the surface age of reddened olivine asteroids, and the surface age of Vesta might be one order of magnitude longer. Since energy efficiency of the space weathering due to real micrometeorite impacts is unknown, this timescale might be the upper limit.

\section{Conclusions}

Using mafic silicates, we performed the proton implantation, the pulse laser irradiation, and the pulse laser irradiation onto proton implanted samples. And we measured reflectance spectra of the altered materials. For the purpose of simulating micrometeorite bombardments, we made a new apparatus using a pulse laser whose pulse length is 6-8 nanoseconds, compatible with the timescale of micrometeorite impacts. We also investigate the influence of the proton implantation on samples. The laser irradiation on olivine causes the largest reduction of albedo and the highest reddening of reflectance spectrum. In general, the variation of olivine is much larger than that of pyroxenes. The proton existence does not influence the spectral change due to the laser irradiation. FeO content of pyroxenes does not relate to the degree of reflectance change. Surface images of the laser irradiated samples suggest that surface alteration would proceed through evaporation rather than melting.

Resulting reflectance spectra after the pulse laser irradiation show not only reduction of albedo but significant reddening, which is observed in asteroids and lunar samples. However, our results show little relative decrease in the ab- sorption band depth ( $1 \mu \mathrm{m}$ for olivine, 1 and $2 \mu \mathrm{m}$ for pyroxene). The obtained spectra are compared with those of olivine asteroids and Vesta. Since olivine is much more easily altered than pyroxene, olivine-rich bodies would be more likely reddened. Our results suggest that Vesta surface is one order of magnitude older than weathered olivine asteroids.

Acknowledgments. The authors appreciate Y. Iijima, K. Shirai, and T. Okada for help in making out apparatus, and T. Kadono and T. Nishimura for maintenance of the laser device. We also thank N. Geshi for his help with preparation of mineral powders and EDS operation, M. Kishima and H. Takayama for help in XRD analysis, and T. Kogure for preliminary analysis by a transmission electron microscope. We wish to thank T. Kawanishi and A. Morita for the RAPID operation, Y. Kuwata for advice of a sample holder, and Y. Hamabe for her helping in proton implantation experiments. We are grateful to M. Otake and J. Owada for the measurements of the reflectance spectra at Tsukuba Space Center of NASDA. We thank J. T. Wasson and H. Takeda for discussions and A. T. Basilevsky for comments. We thank K. Tomeoka and R. E. Johnson for their thoughtful reviews.

\section{References}

Allen, C. C., R. V. Morris, H. V. Lauer, Jr., and D. S. McKay, Microscopic iron metal on glass and minerals-A tool for studying regolith maturity, Icarus, 104, 291-300, 1993.

Chapman, C. R., S-type asteroids, ordinary chondrites, and space weathering: The evidence from Galileo's fly-bys of Gaspra and Ida, Meteorit. Planet. Sci., 31, 699-725, 1996.

Clark, B. E., F. P. Fanale, and J. W. Salisbury, Meteorite-asteroid spectral comparison: The effects comminution, melting, and recrystallization, Icarus, 97, 288-297, 1992.

Dukes, C. A., R. A. Baragiola, and L. A. McFadden, Surface modification of olivine by $\mathrm{H}^{+}$and $\mathrm{He}^{+}$bombardment, J. Geophys. Res., 104, 1865-1872, 1999.

Gaffey, M. J., J. F. Bell, R. H. Brown, T. H. Burbine, J. L. Piatek, K. L. Reed, and D. A. Chaky, Mineralogical variations within the S-Type asteroid class, Icarus, 106, 573-602, 1993.

Grün, E., H. Fechtig, M. S. Hanner, J. Kissel, B.-A. Lindblad, D. Linkert, G. Morfill, and H. A. Zook, In-situ exploration of dust in the solar system and initial results from the Galileo Dust Detector, in Origin and Evolution of Interplanetary Dust, edited by A. C. Levasseur-Regourd and H. Hasegawa, pp. 21-32, Kluwer Academic Press, Dordrecht, 1991.

Hapke, B. Why is the moon dark?, Lunar Planet. Sci., XXIV, 605-606, 1993. 
Helfenstein, P., J. Veverka, P. C. Thomas, D. P. Simonelli, P. Lee, K. Klaasen, T. V. Johnson, H. Brenemen, J. W. Head, S. Murchie, F. Fanale, M. Robinson, B. Clark, J. Granahan, H. Garbeil, A. S. McEwen, R. L. Kirk, M. Davies, G. Neukum, S. Mottola, R. Wagner, M. Belton, C. Chapman, and C. Pilcher, Galileo Photometry of Asteroid 951 Gaspra, Icarus, 107, 37-60, 1994.

Helfenstein, P., J. Veverka, P. C. Thomas, D. P. Simonelli, K. Klaasen, T. V. Johnson, F. Fanale, J. Granahan, A. S. McEwen, M. Belton, and C. Chapman, Galileo Photometry of Asteroid 243 Ida, Icarus, 120, 48-65, 1996.

Hiroi, T. and H. Takeda, Reflectance spectroscopy and mineralogy of primitive achondrites-lodranites, Proc. NIPR Symp. Antarct. Meteorites, 4, 163-177, 1991.

Johnson, R. E. and R. Baragiola, Lunar surface: Sputtering and secondary ion mass spectrometry, Geophys. Res. Lett., 18, 2169-2172, 1991.

Keller, L. P. and D. S. McKay, The nature and origin of rims on lunar soil grains, Geochim. Cosmochim. Acta., 61, 2331-2341, 1997.

Kissel, J. and F. R. Krueger, Ion formation by impact of fast dust particles and comparison with related techniques, Appl. Phys. A, 42, 69-85, 1987.

McCord, T. B., J. B. Adams, and T. V. Johnson, Asteroid Vesta: Spectral reflectivity and compositional implications, Science, 168, 1445-1447, 1970 .

Moroz, L. V., A. V. Fisenko, L. F. Semjonova, C. M. Pieters, and N. N. Korotaeva, Optical effects of regolith processes on S-asteroids as simulated by laser shots on ordinary chondrite and other mafic materials, Icarus, 122, 366-382, 1996.
Ohashi, H., Y. Koizumi, and K. Kobayashi, Carbon-coated film method PIXE for thick and insulating samples, Nucl. Instr. and Meth., B75, 140143, 1993.

Ohtake, M. and H. Ohtake, Change of mineral reflectance spectra caused by melting and reduction, in 29th Annual Lunar and Planetary Science Conference, 1570 pp., 1998.

Pieters, C. M., E. M. Fischer, O. Rode, and A. Basu, Optical effects of space weathering: The role of the finest fraction, J. Geophys. Res., 98, 20817-20824, 1993.

Wasson, J. T., C. M. Pieters, A. V. Fisenko, L. F. Semjonova, and P. H. Warren, Simulation of space weathering of eucrites by laser impulse irradiationm, in 29th Annual Lunar and Planetary Science Conference, 1940 pp., 1998.

Vaniman, D., R. Reedy, G. Heiken, G. Olhoeft, and W. Mendell, The lunar environment, in Lunar Source Book, edited by G. Heiken, D. Vaniman, and B. French, pp. 27-60, Cambridge, 1991.

M. Yamada (e-mail: yamada@jsforum.or.jp), S. Sasaki (e-mail: sho@ geol.s.u-tokyo.ac.jp), H. Nagahara (e-mail: hiroko@geol.s.u-tokyo.ac.jp), A. Fujiwara (e-mail: fujiwara@planeta.sci.isas.ac.jp), S. Hasegawa (e-mail: hasegawa@planeta.sci.isas.ac.jp), H. Yano (e-mail: yano@planeta.sci.isas. ac.jp), T. Hiroi (e-mail: takahiro_hiroi@brown.edu), H. Ohashi (e-mail: ohashi@tokyo-u-fish.ac.jp), H. Otake (e-mail: ootake.hisashi@nasda. go.jp) 